\title{
A Generic Sample Splitting Approach for Refined Community Recovery in Stochastic Block Models
}

\author{
Jing $\mathrm{Lei}^{1}$ and Lingxue $\mathrm{Zhu}^{2}$ \\ ${ }^{1,2}$ Department of Statistics, Carnegie Mellon University
}

November 7, 2014

\begin{abstract}
We propose and analyze a generic method for community recovery in stochastic block models and degree corrected block models. This approach can exactly recover the hidden communities with high probability when the expected node degrees are of order $\log n$ or higher. Starting from a roughly correct community partition given by some conventional community recovery algorithm, this method refines the partition in a cross clustering step. Our results simplify and extend some of the previous work on exact community recovery, discovering the key role played by sample splitting. The proposed method is simple and can be implemented with many practical community recovery algorithms.
\end{abstract}

\section{Introduction}

Stochastic block models (Holland et al., 1983) are a popular tool in modeling the cooccurrence of pairwise interactions between individuals in a population of interest. In recent years, the stochastic block model and its variants, such as the degree corrected block model (Karrer \& Newman, 2011), have been the focus of much research effort in statistics and machine learning, with wide applications in social networks (Faust \& Wasserman, 1992), biological networks and information networks (see, e.g., Kemp et al., 2006; Bickel \& Chen, 2009).

Consider a network data on $n$ nodes recorded in the form of an $n$ by $n$ adjacency matrix $A$, where an entry $A_{i j}=1$ if an interaction is observed between nodes $i$ and $j$, and $A_{i j}=0$ otherwise. The stochastic block model assumes that the nodes are partitioned into $K$ disjoint communities, and that given the community partition, $A_{i j}$ is an independent Bernoulli random variable whose probability only depends on the community membership of $i$ and $j$. Such a model naturally captures the community structure commonly observed 
in network data. It is also possible to incorporate node specific connectivity parameters, and the resulting degree corrected block model allows the network data to have arbitrary degree distribution.

A key inference problem in the stochastic block model and its variants is to recover the hidden communities from an observed adjacency matrix. Various methods have been developed in the last decade, including modularity based methods (Newman \& Girvan, 2004), likelihood methods (Bickel \& Chen, 2009; Zhao et al., 2012), convex optimization (Chen et al., 2012; Le et al., 2014; Abbe et al., 2014), spectral methods (McSherry, 2001; Coja-Oghlan, 2010; Rohe et al., 2011; Jin, 2012; Chaudhuri et al., 2012; Fishkind et al., 2013; Massoulie, 2013; Lei \& Rinaldo, 2013; Vu, 2014), and others (Decelle et al., 2011; Mossel et al., 2013; Anandkumar et al., 2014). These methods are proved to be successful under different assumptions with different types of performance guarantee.

In this paper we propose and analyze a simple and generic sample splitting approach for exact community recovery in both stochastic block models and degree corrected block models. By exact community recovery, we mean that an algorithm can correctly recover the community membership for all nodes with high probability. Our method first randomly splits the nodes into two subsets and obtains a preliminary community recovery on one subset using some conventional algorithm. Then this preliminary community recovery is used to obtain exact recovery for the other subset of nodes in a cross clustering step. We prove that this method can exactly recover the communities with high probability when the expected node degrees are at least $C \log n$ for some constant $C$, and the preliminary community recovery algorithm provides a good approximation to the truth.

Exact recovery for stochastic block models was first studied by McSherry (2001), where a combinatorial projection spectral method was combined with sample splitting. Recently Vu (2014) modified this method to a simpler projection based on singular value decomposition, combined with multiple sample splittings. Chaudhuri et al. (2012) extended the method of McSherry (2001) to a special case of degree corrected block models under stronger assumptions on the edge density. Our results provide further insights to these works by showing that the key ingredient for exact recovery is indeed sample splitting, which can be used in combination with a much broader class of community recovery algorithms. In particular, the simple spectral clustering method, which applies $k$-means to the top eigenvectors of the adjacency matrix, is a popular method with good empirical performance. But there is no theory so far that guarantees its ability of exact recovery. Our result implies that exact recovery can be achieved by combining simple spectral clustering with sample splitting.

There are other methods for exact recovery in stochastic block models. Bickel \& Chen (2009) proved exact recovery for a profile likelihood estimator, for models with node degrees of order $\omega(\log n)$. It is computationally demanding to maximize the profile likelihood. Chen et al. (2012) studied a convex optimization method, which requires $\Omega\left((\log n)^{4}\right)$ ex- 
pected node degrees for exact recovery. In contrast, our theory requires the expected node degrees to be at least $C \log n$ for some constant $C$. This matches the optimal rate established in Abbe et al. (2014), in that exact recovery is impossible when the maximum node degree is less than $c \log n$ for some constant $c$. Moreover, to the best of our knowledge, we give the first exact recovery method for general degree corrected block models with $\Omega(\log n)$ expected node degrees.

\section{Background}

In a stochastic block model, the nodes of a network are partitioned into $K$ disjoint communities. Let $g_{i} \in\{1, \ldots, K\}$ be the community label of node $i$. The observed data is an $n \times n$ symmetric binary random matrix $A$ with independent upper-diagonal entries $A_{i j}$ $(1 \leq i<j \leq n): P\left(A_{i j}=1\right)=1-P\left(A_{i j}=0\right)=B_{g_{i} g_{j}}$, where $B \in[0,1]^{K \times K}$ is a symmetric matrix representing the community-wise edge probabilities. For convenience we assume $A_{i i}=0$ for all $i$. Throughout this paper we assume that $K$, the number of communities, is known.

The community recovery problem concerns estimating the membership vector $g=\left(g_{1}, \ldots, g_{n}\right)$, up to a label permutation. It is well-known that the hardness of community recovery depends on (i) the sample size $n$, (ii) number of communities $K$, (iii) differences between the rows of $B$, and (iv) the magnitude of the entries in $B$, which controls the overall density of edges in the observed network. In most theoretical studies of community recovery, it is common practice to consider the large sample behavior where $n$ grows to infinity, while other model parameters, such as $K$ and $B$, change as functions of $n$. In the current paper, for simplicity we focus on the network edge density, and fix other parameters as constants. Our analysis can be used to investigate dependence on other model parameters, as discussed in Section 5. To this end, we assume that

$$
B=\alpha_{n} B_{0},
$$

where $B_{0}$ is a $K \times K$ non-negative symmetric constant matrix with maximum entry 1 . In Abbe et al. (2014) it is shown that, for a special class of stochastic block models where $K=2$ and $B_{0}(1,1)=B_{0}(2,2)=1$, exact recovery is possible if and only if $\alpha_{n}>C \log n / n$ for a constant $C$ depending on the off-diagonal entry $B_{0}(1,2)$. For general stochastic block models, McSherry (2001) studied a spectral method with exact recovery when $\alpha_{n}=$ $\Omega\left((\log n)^{3.5} / n\right)$. Vu (2014) improved this result to $\alpha_{n} \geq C \log n / n$ for a sufficiently large $C$. Other methods, such as convex optimization (Chen et al., 2012) and profile likelihood (Bickel \& Chen, 2009), require stronger conditions on $\alpha_{n}$.

The algorithm proposed by McSherry (2001) first randomly splits the columns of $A$ into two sets. Then each set of columns are projected onto a $K$ dimensional subspace constructed 


\section{Algorithm 1: Cross Clustering (CrossClust)}

Input: adjacency matrix $A$; subset of nodes $\mathcal{V}_{1}$; subset of nodes $\mathcal{V}_{2}$; membership vector $\hat{g}^{(1)}$ on $\mathcal{V}_{1}$.

Require subroutine: distance based clustering algorithm $\mathcal{D}$.

1. For each $v \in \mathcal{V}_{2}$, let $\hat{h}_{v} \leftarrow\left(\hat{h}_{v, 1}, \ldots, \hat{h}_{v, K}\right)$ with

$$
\hat{h}_{v, k} \leftarrow \frac{\sum_{v^{\prime} \in \mathcal{V}_{1}, g_{v^{\prime}}^{(1)}=k} A_{v, v^{\prime}}}{\#\left\{v^{\prime}: v^{\prime} \in \mathcal{V}_{1}, g_{v^{\prime}}^{(1)}=k\right\}} .
$$

2. Output $\hat{g}^{(2)} \leftarrow \mathcal{D}\left(\left\{\hat{h}_{v}: v \in \mathcal{V}_{2}\right\}, K\right)$.

from the other set. The final community recovery is given by applying a clustering algorithm to the $n \times n$ matrix obtained after the column projection. In the main algorithm analyzed in that paper, the $K$ dimensional subspaces are obtained using a combinatorial approach called CProj, which is more complicated than some natural choices such as singular value decomposition. $\mathrm{Vu}$ (2014) simplified the CProj subroutine by using multiple random splits and projection onto singular subspaces of the split matrices. Although this method gives stronger theoretical guarantees, the procedure remains somehow cumbersome and it is not clear how the random splitting and low dimensional projection work together to give exact recovery.

In this paper we argue that a single sample splitting can lead to exact recovery under a much broader context. In particular, one can start from any community recovery algorithm with approximate recovery guarantee, and use a single sample splitting to refine the output to achieve exact recovery. The key idea is that once we have a roughly correct community membership for a subset of the nodes, it can be used to produce exact recovery for the remaining nodes. This is described in detail in Algorithm 1.

The intuition behind Algorithm 1 is very natural. Suppose we have approximately correct memberships for nodes in $\mathcal{V}_{1}$. Then we can use this membership to estimate the communitywise edge probability for each node in $\mathcal{V}_{2}$. For $v \in \mathcal{V}_{2}$ we must have

$$
\hat{h}_{v, k} \approx B_{g_{v}, k}, \quad k=1, \ldots, K .
$$

Therefore, if two nodes $v$ and $v^{\prime}$ are in the same community, then their corresponding $\hat{h}$ vectors shall both be close to the same row of $B$. This gives us a good embedding of these nodes in a $K$ dimensional Euclidean space with a nearly perfect clustering structure. If such an embedding is good enough, for example, the distance between any two cluster centers is at least four times larger than the distance between any point to its center, then, as pointed out in $\mathrm{Vu}$ (2014), some classical distance-based clustering algorithms such as minimum spanning tree can perfectly recover the communities. 
Algorithm 2: Community Recovery by Sample Splitting (SplitClust)

Input: adjacency matrix $A$; number of communities $K$.

Require subroutine: CrossClust; initial community recovery algorithm $\mathcal{S}$.

1. Randomly split the nodes into two equal sized sets, $\mathcal{V}_{1}$ and $\mathcal{V}_{2}$.

2. $\hat{g}^{(1)} \leftarrow \mathcal{S}\left(A^{(1)}, K\right)$, where $A^{(1)}$ is the induced adjacency matrix over $\mathcal{V}_{1}$.

3. $\hat{g}^{(2)} \leftarrow \operatorname{Cross} \operatorname{Clust}\left(A, \mathcal{V}_{1}, \mathcal{V}_{2}, \hat{g}^{(1)}\right)$.

4. Output $\hat{g} \leftarrow \operatorname{Cross} C l u s t ~\left(A, \mathcal{V}_{2},[n], \hat{g}^{(2)}\right)$.

This algorithm also reveals the need for sample splitting. In the beginning, it may seem natural to obtain an approximate recovery for the entire set of nodes and calculate the $K$-dimensional embedding for each node based on this community partition. This method, although may work well in practice, is hard to analyze. The complex dependence between

the numerator and denominator in the definition of $\hat{h}_{v, k}$ makes it highly challenging to analyze the error of $\hat{h}_{v, k}$ as an estimator of $B_{g_{v}, k}$. In contrast, sample splitting introduces more independence between these two terms and makes the analysis much more tractable.

Our main algorithm for stochastic block models based on sample splitting is described in Algorithm 2. The initial community recovery algorithm $\mathcal{S}$ can be chosen by the user. As shown in Section 3 below, it only needs to satisfy some mild accuracy requirement, which can be achieved by many popular and practical methods, such as spectral methods and likelihood based methods.

\section{Main results for stochastic block models}

Terminology Throughout this paper, the term "with high probability" means "with probability at least $1-O\left(n^{-1}\right)$ ". For two community membership vectors $g$ and $\hat{g}$, we say $\hat{g}$ makes $m$ recovery errors as an estimate of $g$, where $m$ is the smallest integer such that there exists a label permutation on $\hat{g}$ under which $\hat{g}$ and $g$ disagree at exactly $m$ entries. We write $\hat{g}=g$ if $\hat{g}$ makes zero error. For a membership vector $g$ on $[n]$, and a subset $\mathcal{V} \subseteq[n], g^{(\mathcal{V})}$ denotes the membership vector obtained by confining $g$ on $\mathcal{V}$. We use $g^{(j)}$ in place of $g^{\left(\mathcal{V}_{j}\right)}(j=1,2)$ for simplicity. We use $\|\cdot\|$ to denote the $\ell_{2}$ norm of vectors in Euclidean spaces. For any matrix $A$, we refer to its $(i, j)$ th element as $A(i, j)$, and its $i$-th row as $A(i, \cdot)$. Sometimes $A_{i, j}$ is used in place of $A(i, j)$ for brevity.

To focus on the network edge sparsity, we assume that the smallest community size is proportional to $n$.

Definition 1 (Proper membership). Given a subset $\mathcal{V} \subseteq[n]$, a membership vector $g$ on $\mathcal{V}$, and a positive constant $\pi_{0} \in(0,1 / K]$, we say $g^{(\mathcal{V})}$ is $\pi_{0}$-proper if $\min _{1 \leq k \leq K} \mid\left\{i \in \mathcal{V}: g_{i}=\right.$ 
$k\} \mid \geq \pi_{0} n$

We make the following assumptions.

(A1) In the network sparsity scaling in (1), $B_{0}$ is a constant matrix such that the minimum $\ell_{2}$ difference between two rows of $B_{0}$ is at least $\gamma$, a positive constant.

(A2) The true community membership $g$ on $\mathcal{V}=[n]$ is $\pi_{0}$-proper for some constant $\pi_{0}>0$.

(A3) The initial community recovery algorithm $\mathcal{S}$, with high probability, has recovery error at most $n / f\left(n \alpha_{n}\right)$ when $\alpha_{n}=\Omega(\log n / n)$, where $f$, possibly depending on $\pi_{0}$ and $B_{0}$, satisfies $\lim _{x \rightarrow \infty} f(x) \uparrow \infty$.

Assumption A1 puts a lower bound on pairwise difference between the rows of $B_{0}$, which is a minimum requirement for the communities to be distinguishable. This also implies that $K$ is a fixed constant. Assumption A2 puts a lower bound on the minimum community size. These are mainly for simplicity of presentation, so that we can focus on the dependence on the network sparsity. Our argument does allow for some mild generalizations so that both the number of communities and the minimum community size can change with $n$ in a non-trivial manner, as discussed in Section 5. Assumption A3 puts a mild requirement on the accuracy of the initial community recovery algorithm. That is, with high probability the initial algorithm $\mathcal{S}$ correctly recovers the membership of all but a vanishing proportion of nodes, as the expected node degrees grow at $\Omega(\log n)$ rate or faster. It can be satisfied by some very simple and practical methods. For example, the simple spectral clustering method, which applies $k$-means to the rows of leading eigenvectors of the adjacency matrix, satisfies Assumption A3 with $f\left(n \alpha_{n}\right)=L\left(\pi_{0}, B_{0}\right) n \alpha_{n}$ for some function $L>0$ independent of $n$ when $B_{0}$ has full rank (Lei \& Rinaldo, 2013). Our theoretical developments will focus on the case where $n$ is large enough such that $f\left(n \alpha_{n}\right)$ dominates any constant quantities involved in the analysis.

In the following analysis, we consider Algorithms 1 and 2 with subroutine $\mathcal{D}$ being minimum spanning tree clustering, which constructs a minimum spanning tree on the input data and removes the $K-1$ largest edges. We have the following performance guarantee of Algorithm 1.

Theorem 2 (Exact recovery using sample splitting). Given a membership vector $g$ and connectivity matrix $B=\alpha_{n} B_{0}$ satisfying Assumptions A1, A2, let $\hat{g}$ be the output of SplitClust (Algorithm 2) with input matrix $A$ generated by the corresponding stochastic block model and subroutine $\mathcal{S}$ satisfying Assumption A3. There exists a constant $C$ such that if $\alpha_{n} \geq C \log n / n$ then with high probability we have $\hat{g}=g$.

Theorem 2 essentially says that when the true community partition is balanced and the expected node degrees are sufficiently larger than $\log n$, one can always use sample splitting to refine an approximately correct community recovery algorithm to achieve exact recovery. 
The proof, given in Section 6.1, consists of two simple applications of the key result Lemma 3 below, which guarantees the accuracy of subroutine CrossClust.

Lemma 3 (Accuracy of CrossClust). Let $A$ be an adjacency matrix generated by a stochastic block model satisfying the assumptions of Theorem 2 , and $\mathcal{V}_{1}$ be a subset with $\pi_{0}$-proper membership vector $g^{(1)}$. Let $\hat{g}^{(1)}$ be an estimated membership vector on $\mathcal{V}_{1}$ independent of the edges between $\mathcal{V}_{1}$ and $\mathcal{V}_{2}$, with recovery error at most $\left|\mathcal{V}_{1}\right| / f\left(\left|\mathcal{V}_{1}\right| \alpha_{n}\right)$. There exists a constant $C$ such that if $\alpha_{n} \geq C \log n / n$, then with high probability, $\hat{g}^{(2)}$, the output of Algorithm 1, satisfies $\hat{g}^{(2)}=g^{(2)}$.

Lemma 3 ensures that the subroutine CrossClust produces exact recovery on $\mathcal{V}_{2}$ with high probability. The probabilistic claim in Lemma 3 is indeed conditional on given $\hat{g}^{(1)}$. Here we do not emphasize the conditional nature of this result as the randomness is from edges between $\mathcal{V}_{1}$ and $\mathcal{V}_{2}$, and hence is independent of $\hat{g}^{(1)}$ by assumption. The proof of Lemma 3 , as detailed in Section 6.1, is based on a careful decomposition of estimation error $\mid \hat{h}_{v, k}-$ $B\left(g_{v}, k\right) \mid$ followed by large deviation bounds.

\section{Extension to degree corrected block models}

The degree corrected block model (Karrer \& Newman, 2011) extends the stochastic block model by introducing additional node level degree heterogeneity. In addition to the membership vector $g$ and community-wise connectivity matrix $B$, the degree corrected block model incorporates a parameter $\psi \in(0,1]^{n}$ to model the node level activeness. Then the edge $A_{i j}$ between nodes $i$ and $j$ is an independent Bernoulli variable with parameter $\psi_{i} \psi_{j} B_{g_{i} g_{j}}$. For identifiability, we assume $\max _{i: g_{i}=k} \psi_{i}=1$, for all $1 \leq k \leq K$. The parameter $\psi_{i}$ reflects the relative activeness of node $i$ in its community. The degree corrected block model is able to model a much wider range of network data and is more realistic than the regular stochastic block model. There are relatively fewer results on exact recovery for degree corrected block models. Zhao et al. (2012) extended the result of Bickel \& Chen (2009), showing that the profile likelihood estimator can recover exactly when $\alpha_{n}=\omega(\log n / n)$. Chaudhuri et al. (2012) extended the method of McSherry (2001) to a special case of degree corrected models with a stronger requirement on the decay rate of $\alpha_{n}$. In the following we show that the simple sample splitting method can be successful under general degree corrected block models when $\alpha_{n} \geq C \log n / n$ for sufficiently large constant $C$.

Under the degree corrected block model, we need to modify the CrossClust algorithm so that the effect of nuisance parameter $\psi$ is cancelled out by a normalization step. To this end, we introduce the spherical cross clustering algorithm in Algorithm 1'.

The exact recovery property of the sample splitting approach can be established for degree 
Algorithm 1': Spherical Cross Clustering (CrossClustSphere)

Input: adjacency matrix $A$; subset of nodes $\mathcal{V}_{1}$; subset of nodes $\mathcal{V}_{2}$; membership vector $g^{(1)}$ on $\mathcal{V}_{1}$.

Require subroutine: distance based clustering algorithm $\mathcal{D}$.

1. For each $v \in \mathcal{V}_{2}$, let $\hat{h}_{v}=\left(\hat{h}_{v, 1}, \ldots, \hat{h}_{v, K}\right)$ be the same as give in Step 1 of Algorithm 1 (CrossClust).

2. Output $\hat{g}^{(2)} \leftarrow \mathcal{D}\left(\left\{\hat{h}_{v} /\left\|\hat{h}_{v}\right\|: v \in \mathcal{V}_{2}\right\}, K\right)$.

corrected block models under essentially the same conditions as for the regular stochastic block models, with a modified community separation condition and an additional condition on the nuisance parameter $\psi$. Recalling that $B_{0}(k, \cdot)$ denotes the $k$ th row of $B_{0}$, we assume the following.

(A1') The minimum $\ell_{2}$ difference between two normalized-rows of $B_{0}$ is at least $\tilde{\gamma}$, a positive constant:

$$
\min _{1 \leq k<k^{\prime} \leq K}\left\|\frac{B_{0}(k, \cdot)}{\left\|B_{0}(k, \cdot)\right\|}-\frac{B_{0}\left(k^{\prime}, \cdot\right)}{\left\|B_{0}\left(k^{\prime}, \cdot\right)\right\|}\right\| \geq \tilde{\gamma}>0 .
$$

(A4) $\min _{1 \leq i \leq n} \psi_{i} \geq \psi_{0}$ for some constant $\psi_{0} \in(0,1]$.

Assumption A1' modifies Assumption A1 to account for the normalization step in CrossClustSphere, which is necessary for degree corrected block models because two rows in $B$ differing only by a constant scaling are indistinguishable due to the node activeness parameter. Assumption A4 prevents any node from being too inactive, otherwise there will be too few edges for that node, making exact recovery unlikely. Under these assumptions, the spherical spectral clustering method described and analyzed in Lei \& Rinaldo (2013) satisfies Assumption A3 with $f\left(n \alpha_{n}\right)=L^{\prime}\left(\pi_{0}, B_{0}\right) \sqrt{n \alpha_{n}}$, provided that $B_{0}$ has full rank.

Theorem 4 (Exact recovery for degree corrected block models). Let $A$ be an adjacency matrix generated from a degree corrected block model with membership vector $g$, connectivity matrix $B=\alpha_{n} B_{0}$, and node activeness vector $\psi$ satisfying Assumptions A1', A2 and A4. Let $\hat{g}$ be the output of SplitClust (Algorithm 2) using subroutine CrossClustSphere (Algorithm 1') instead of CrossClust and initial recovery algorithm $\mathcal{S}$ satisfying Assumption A3. There exists a constant $C$ such that if $\alpha_{n} \geq C \log n / n$ then $\hat{g}=g$ with high probability.

The proof of Theorem 4 is similar to that of Theorem 2, and uses the following analogous result of Lemma 3. Detailed proofs of both results are given in Section 6.2.

Lemma 5 (Accuracy of CrossClustSphere). Let $A$ be an adjacency matrix generated by a degree corrected block model satisfying the assumptions of Theorem 4 , and $\mathcal{V}_{1}$ be a subset with $\pi_{0}$-proper membership vector $g^{(1)}$. Let $\hat{g}^{(1)}$ be a membership vector on $\mathcal{V}_{1}$ independent 
of the edges between $\mathcal{V}_{1}$ and $\mathcal{V}_{2}$, with recovery error at most $\left|\mathcal{V}_{1}\right| / f\left(\left|\mathcal{V}_{1}\right| \alpha_{n}\right)$. There exists a constant $C$ such that if $\alpha_{n} \geq C \log n / n$, then with high probability, $\hat{g}^{(2)}$, the output of CrossClustSphere (Algorithm 1'), satisfies $\hat{g}^{(2)}=g^{(2)}$.

\section{Discussion}

Dependence on other model parameters. In this paper we investigate exact community recovery with a special focus on the overall network sparsity (e.g., the rate at which $\alpha_{n}$ vanishes). In the study of stochastic block models, the effect of other model parameters, such as the number of communities and community size imbalance may also be of interest. The arguments developed in this paper, for example, Lemma 6, do keep track of these parameters and hence can be used to study the more general scenario where these parameters are also allowed to change with $n$ non-trivially. In particular, when $K=2$ with $\mathcal{I}_{1}, \mathcal{I}_{2}$ being the two communities, one can show that the sample splitting approach succeeds with high probability when $\alpha_{n}=\Omega(1)$ and $\min \left(\left|\mathcal{I}_{1}\right|,\left|\mathcal{I}_{2}\right|\right) \geq C \sqrt{n}$ for large enough $C$.

Extension to V-fold cross clustering Our main algorithm, Algorithm 2, splits the nodes into two subsets. Practically, halving the set of nodes may result in a non-negligible loss of accuracy in the initial estimation of $g^{(1)}$. This issue can be mitigated by extending the sample splitting to a $\mathrm{V}$-fold cross clustering. Given positive integer $V \geq 2$, we can split the nodes into $V$ subsets, $\mathcal{V}_{1}, \ldots, \mathcal{V}_{V}$ of equal size. For each $j=1, \ldots, V$, let $\mathcal{V}_{-j}=\cup_{j^{\prime} \neq j} \mathcal{V}_{j^{\prime}}$. Then we can obtain a preliminary community estimate $\hat{g}^{(-j)}$ for $\mathcal{V}_{-j}$, and then apply CrossClust to $\left(A, \mathcal{V}_{-j}, \mathcal{V}_{j}, \hat{g}^{(-j)}\right)$ to obtain community estimate $\hat{g}^{(j)}$ for $\mathcal{V}_{j}$. Our theoretical results can be extended to this case, suggesting that $\hat{g}^{(j)}=g^{(j)}$ with high probability under appropriate conditions. After obtained $\hat{g}^{(j)}$ for all $1 \leq j \leq V$, one can merge these membership vectors by matching the edge frequencies between communities in different subsets. It has been empirically observed that such $\mathrm{V}$-fold cross clustering with $V>2$ usually outperforms the sample splitting method.

Open problems The only step in our proof that requires sample splitting is the large deviation bound for the term $T_{1}$ in the proofs of Lemmas 6 and 9, where the summation of $A_{v, v^{\prime}}$ is over a random set $\left\{v^{\prime} \in \mathcal{V}_{1}: \hat{g}_{v^{\prime}}^{(1)} \neq g_{v^{\prime}}^{(1)}\right\}$. The sample splitting makes the summand $A_{v, v^{\prime}}$ independent of this index set, allowing us to condition on the index set and apply Bernstein's inequality. As mentioned earlier in Section 2, a natural alternative is to obtain a preliminary community estimate for the entire set of nodes, and then cross cluster each node using this preliminary community partition. That is, we use $\mathcal{V}_{1}=\mathcal{V}_{2}=[n]$ in Algorithm 1. Such a self-cross clustering approach, although hard to analyze, gives very competitive practical performance. As an example, consider the political blog data 
(Adamic \& Glance, 2005), where the edges represent hyperlinks among 1222 weblogs on U.S. politics in 2004, and two communities are recognized as "liberal" and "conservative", respectively. It is widely believed that the degree corrected block model with $K=2$ fits the data well (Zhao et al., 2012; Jin, 2012; Yan et al., 2014). The self-cross clustering method makes about $10 \%$ fewer errors than both the simple spherical spectral clustering (Lei \& Rinaldo, 2013) and the V-fold cross clustering considered in this paper. It would be an interesting open problem to provide rigorous performance guarantee for the self-cross clustering method.

\section{Proof of main results}

We introduce several more notations. For any $i \in\{1,2\}, k \in\{1,2, \ldots, K\}$, we denote

$$
\mathcal{I}_{k}=\left\{v: g_{v}=k\right\}, \mathcal{I}_{k}^{(i)}=\left\{v: v \in \mathcal{V}_{i}, g_{v}^{(i)}=k\right\}, \hat{\mathcal{I}}_{k}^{(i)}=\left\{v: v \in \mathcal{V}_{i}, \hat{g}_{v}^{(i)}=k\right\}
$$

Usually the true membership $g$ and estimated membership $\hat{g}$ agree on most entries up to a label permutation. For simplicity we will assume, without loss of generality, that the permutation is identity.

\subsection{Proofs for stochastic block models}

The main theorem for stochastic block models follows from two simple applications of the accuracy of cross clustering (Lemma 3).

Proof of Theorem 2. Note that

$$
\mathbb{P}(\hat{g}=g) \geq \mathbb{P}\left(\hat{g}=g, \hat{g}^{(2)}=g^{(2)}\right)=\mathbb{P}\left(\hat{g}=g \mid \hat{g}^{(2)}=g^{(2)}\right) \mathbb{P}\left(\hat{g}^{(2)}=g^{(2)}\right) .
$$

By Lemma $7, g^{(1)}$ is $\pi_{0} / 3$-proper with high probability. Then by Lemma 3 , when $\alpha_{n} \geq$ $C \log n / n$ for some constant $C, \hat{g}^{(2)}=g^{(2)}$ with high probability.

Finally, when $\hat{g}^{(2)}=g^{(2)}, \hat{g}^{(2)}$ is $\pi_{0} / 3$-proper with high probability according to Lemma 7 . Then by Lemma 3 again, $\hat{g}=g$ with high probability when $\alpha_{n}>C^{\prime} \log n / n$ for another large enough constant $C^{\prime}$.

Proof of Lemma 3. Suppose $\alpha_{n}=a \log n / n$ for some constant $a$. If for all nodes $v \in \mathcal{V}_{2}$, for some constant $\delta>0$,

$$
\left\|\hat{h}_{v}-B\left(g_{v}, \cdot\right)\right\| \leq \delta \frac{\log n}{n}
$$


then we have the following separation conditions

$$
\begin{aligned}
& \sup _{v, v^{\prime} \in \mathcal{V}_{2}, g_{v}=g_{v^{\prime}}}\left\|\hat{h}_{v}-\hat{h}_{v^{\prime}}\right\| \leq 2 \delta \frac{\log n}{n}, \\
& \inf _{v, v^{\prime} \in \mathcal{V}_{2}, g_{v} \neq g_{v^{\prime}}}\left\|\hat{h}_{v}-\hat{h}_{v^{\prime}}\right\| \geq \inf _{1 \leq k<k^{\prime} \leq K}\left\|B(k, \cdot)-B\left(k^{\prime}, \cdot\right)\right\|-2 \delta \frac{\log n}{n} \geq(a \gamma-2 \delta) \frac{\log n}{n} .
\end{aligned}
$$

The distance based clustering subroutine $\mathcal{D}$ used in Algorithm 1, such as the minimum spanning tree, can correctly cluster all nodes, i.e., $\hat{g}^{(2)}=g^{(2)}$, if

$$
\sup _{v, v^{\prime} \in \mathcal{V}_{2}, g_{v}=g_{v^{\prime}}}\left\|\hat{h}_{v}-\hat{h}_{v^{\prime}}\right\|<\inf _{v, v^{\prime} \in \mathcal{V}_{2}, g_{v} \neq g_{v^{\prime}}}\left\|\hat{h}_{v}-\hat{h}_{v^{\prime}}\right\| .
$$

Therefore, it suffices to show that with high probability, $\left\|\hat{h}_{v}-B\left(g_{v}, \cdot\right)\right\| \leq \delta \frac{\log n}{n}$ for all $v \in \mathcal{V}_{2}$, where

$$
0<\delta<\frac{a \gamma}{4}
$$

By Lemma 6, the approximation bound (2) and inequality (3) hold with high probability if we choose $q$ in Lemma 6 to be a constant larger than $4 / \gamma$.

The following lemma establishes our key observation that $\hat{h}_{v}$ should be close to the corresponding row of connectivity matrix, $B\left(g_{v}, \cdot\right)$.

Lemma 6. Given $\mathcal{V}_{1}, \mathcal{V}_{2}, g^{(1)}$, and $\hat{g}^{(1)}$ satisfying the conditions of Lemma 3 with $\alpha_{n}=$ $q \delta \log n / n$. For any $q>1$, there exists $\delta_{0}=\delta_{0}\left(\pi_{0}, c_{0}, K, q\right)$, such that for all $\delta \geq \delta_{0}$

$$
\mathbb{P}\left(\left\|\hat{h}_{v}-B\left(g_{v}, \cdot\right)\right\| \leq \delta \frac{\log n}{n}, \quad \forall v \in \mathcal{V}_{2}\right)=1-O\left(n^{-1}\right),
$$

where the probability is conditional on $\mathcal{V}_{1}, \mathcal{V}_{2}$ and $\hat{g}^{(1)}$.

Proof. Let $a=q \delta$. Because

$$
\mathbb{P}\left(\left\|\hat{h}_{v}-B\left(g_{v}, \cdot\right)\right\| \geq \delta \frac{\log n}{n}\right) \leq \sum_{k=1}^{K} \mathbb{P}\left(\left|\hat{h}_{v, k}-B\left(g_{v}, k\right)\right| \geq \frac{\delta}{\sqrt{K}} \frac{\log n}{n}\right),
$$

it suffices to bound all $K$ coordinates individually. Now, for any $k \in\{1,2, . ., K\}$, since $\lim _{n \rightarrow \infty} f\left(\pi_{0} a \log n\right)=\infty$, when $n$ is large enough, we have

$$
\begin{aligned}
& \sum_{l \neq k}\left|\hat{\mathcal{I}}_{l}^{(1)} \cap \mathcal{I}_{k}^{(1)}\right| \leq\left|\left\{v^{\prime}: \hat{g}_{v^{\prime}}^{(1)} \neq g_{v^{\prime}}^{(1)}\right\}\right| \leq \frac{\left|\mathcal{V}_{1}\right|}{f\left(\left|\mathcal{V}_{1}\right| \alpha_{n}\right)} \leq \frac{n}{f\left(\pi_{0} a \log n\right)} . \\
& \left|\hat{\mathcal{I}}_{k}^{(1)}\right| \geq\left|\mathcal{I}_{k}^{(1)}\right|-\sum_{l \neq k}\left|\hat{\mathcal{I}}_{l}^{(1)} \cap \mathcal{I}_{k}^{(1)}\right| \geq\left[\pi_{0}-\frac{1}{f\left(\pi_{0} a \log n\right)}\right] n \geq \pi_{0} n / 2 .
\end{aligned}
$$


For any $1 \leq k \leq K$, we have

$$
\begin{aligned}
\left|\hat{h}_{v, k}-B\left(g_{v}, k\right)\right| \leq & \left|\frac{\sum_{v^{\prime} \in \hat{\mathcal{I}}_{k}^{(1)}} A_{v, v^{\prime}}-\sum_{v^{\prime} \in \mathcal{I}_{k}^{(1)}} A_{v, v^{\prime}}}{\left|\hat{\mathcal{I}}_{k}^{(1)}\right|}\right|+\left|\frac{\sum_{v^{\prime} \in \mathcal{I}_{k}^{(1)}} A_{v, v^{\prime}}}{\left|\hat{\mathcal{I}}_{k}^{(1)}\right|}-\frac{\sum_{v^{\prime} \in \mathcal{I}_{k}^{(1)}} A_{v, v^{\prime}}}{\left|\mathcal{I}_{k}^{(1)}\right|}\right| \\
& +\left|\frac{\sum_{v^{\prime} \in \mathcal{I}_{k}^{(1)}} A_{v, v^{\prime}}}{\left|\mathcal{I}_{k}^{(1)}\right|}-B\left(g_{v}, k\right)\right| \\
\leq & \frac{\left|\sum_{v^{\prime} \in \hat{\mathcal{I}}_{k}^{(1)}} A_{v, v^{\prime}}-\sum_{v^{\prime} \in \mathcal{I}_{k}^{(1)}} A_{v, v^{\prime}}\right|}{\pi_{0} n / 2}+\frac{|| \mathcal{I}_{k}^{(1)}|-| \hat{\mathcal{I}}_{k}^{(1)}||}{\pi_{0}^{2} n^{2} / 2} \sum_{v^{\prime} \in \mathcal{I}_{k}^{(1)}} A_{v, v^{\prime}} \\
& +\left|\frac{\sum_{v^{\prime} \in \mathcal{I}_{k}^{(1)}} A_{v, v^{\prime}}}{\left|\mathcal{I}_{k}^{(1)}\right|}-B\left(g_{v}, k\right)\right| \\
= & T_{1}+T_{2}+T_{3} .
\end{aligned}
$$

Thus

$$
\mathbb{P}\left(\left|\hat{h}_{v, k}-B\left(g_{v}, k\right)\right| \geq \frac{\delta}{\sqrt{K}} \frac{\log n}{n}\right) \leq \sum_{j=1}^{3} \mathbb{P}\left(T_{j} \geq \frac{\delta}{3 \sqrt{K}} \frac{\log n}{n}\right) .
$$

Now we only need to bound the three terms individually. First by the assumption on $\hat{g}^{(1)}$ we know $\left|\left\{v^{\prime}: \hat{g}_{v^{\prime}}^{(1)} \neq g_{v^{\prime}}^{(1)}\right\}\right| \leq n / f\left(\pi_{0} a \log n\right)$. Then using Bernstein's inequality we have for $n$ large enough,

$$
\begin{aligned}
\mathbb{P}\left(T_{1} \geq \frac{\delta}{3 \sqrt{K}} \frac{\log n}{n}\right) & \leq \mathbb{P}\left(\sum_{v^{\prime}: \hat{g}_{v^{\prime}}^{(1)} \neq g_{v^{\prime}}^{(1)}} A_{v, v^{\prime}} \geq \frac{\pi_{0} n}{2} \frac{\delta}{3 \sqrt{K}} \frac{\log n}{n}\right) \\
& \leq n^{-\frac{c_{1}^{2}\left(\pi_{0}, K\right) \delta^{2}}{a / f\left(\pi_{0} a \log n\right)+c_{1}\left(\pi_{0}, K\right) \delta / 3}} \leq n^{-c_{1}\left(\pi_{0}, K\right) \delta},
\end{aligned}
$$

where $c_{1}\left(\pi_{0}, K\right)$ is a positive constant depending only on $\pi_{0}$ and $K$.

To control $T_{2}$, similarly we have for $n$ large enough using Bernstein's inequality

$$
\begin{aligned}
\mathbb{P}\left(T_{2}\right. & \left.\geq \frac{\delta}{3 \sqrt{K}} \frac{\log n}{n}\right) \leq \mathbb{P}\left(\sum_{v^{\prime} \in \mathcal{I}_{k}^{(1)}} A_{v, v^{\prime}} \geq \frac{\pi_{0}^{2} n^{2}}{2|| \mathcal{I}_{k}^{(1)}|-| \hat{\mathcal{I}}_{k}^{(1)}||} \frac{\delta}{3 \sqrt{K}} \frac{\log n}{n}\right) \\
& \leq \mathbb{P}\left(\sum_{v^{\prime} \in \mathcal{I}_{k}^{(1)}} A_{v, v^{\prime}} \geq \frac{\pi_{0}^{2} \delta \log n f\left(\pi_{0} a \log n\right)}{6 \sqrt{K}}\right) \\
& \leq n^{-\frac{c_{2}^{2}\left(\pi_{0}, K\right) f^{2}\left(\pi_{0} a \log n\right) \delta^{2}}{a+c_{2}\left(\pi_{0}, K\right) f\left(\pi_{0} a \log n\right) \delta / 3}} \leq n^{-c_{2}\left(\pi_{0}, K\right) \delta},
\end{aligned}
$$


where $c_{2}\left(\pi_{0}, K\right)$ is another positive constant function of $\pi_{0}$ and $K$.

Directly applying Bernstein's inequality to $T_{3}$, we have

$$
\begin{aligned}
\mathbb{P}\left(T_{3} \geq \frac{\delta}{3 \sqrt{K}} \frac{\log n}{n}\right) & \leq \mathbb{P}\left(\left|\sum_{v^{\prime} \in \mathcal{I}_{k}^{(1)}}\left[A_{v, v^{\prime}}-B\left(g_{v}, k\right)\right]\right| \geq \frac{\delta}{3 \sqrt{K}} \frac{\log n}{n} \pi_{0} n\right), \\
& \leq 2 n^{-c_{3}\left(\pi_{0}, K, q\right) \delta},
\end{aligned}
$$

where $c_{3}\left(\pi_{0}, K, q\right)$ is a positive constant function independent of $n$.

The claimed result follows by choosing $\delta$ large enough and applying union bound over all $v \in \mathcal{V}_{2}$.

We conclude this subsection by establishing probability lower bounds of having proper subsets under random splitting.

Lemma 7 (Probability of having proper split subsets). If the true membership vector $g$ on $[n]$ is $\pi_{0}$ proper, and $[n]$ is randomly split into two equal-sized subsets $\mathcal{V}_{1}, \mathcal{V}_{2}$ with corresponding $g^{(1)}, g^{(2)}$. Then $g^{(1)}$ and $g^{(2)}$ are $\pi_{0} / 3$-proper with high probability.

Proof. By definition we have $\left|\mathcal{V}_{1}\right|=\left|\mathcal{V}_{2}\right| \geq n / 2$. The claimed result follows easily from an exponential tail probability bound for hypergeometric random variables (see, e.g., Skala, 2013), for $i=1,2$,

$$
\mathbb{P}\left(\left|\mathcal{I}_{k}^{(i)}\right|<\pi_{0} n / 3\right) \leq \mathbb{P}\left(\left|\mathcal{I}_{k}^{(i)}\right|-\mathbb{E}\left|\mathcal{I}_{k}^{(i)}\right|<-\pi_{0} n / 6\right) \leq e^{-\pi_{0}^{2} n / 18}
$$

\subsection{Proofs for degree corrected block models}

In the following proofs, we denote $\tilde{B}$ as the $K \times K$ weighted connectivity matrix, where

$$
\tilde{B}(i, j)=\frac{\sum_{v^{\prime} \in \mathcal{I}_{j}^{(1)}} \psi_{v^{\prime}}}{\left|\mathcal{I}_{j}^{(1)}\right|} B(i, j) .
$$

Proof of Theorem 4. Note that

$$
\mathbb{P}(\hat{g}=g) \geq \mathbb{P}\left(\hat{g}=g, \hat{g}^{(2)}=g^{(2)}\right)=\mathbb{P}\left(\hat{g}=g \mid \hat{g}^{(2)}=g^{(2)}\right) \mathbb{P}\left(\hat{g}^{(2)}=g^{(2)}\right) .
$$


By Lemma $7, g^{(1)}$ is $\pi_{0} / 3$-proper with high probability. Then by Lemma 5 , when $\alpha_{n}>$ $C \log n / n$ for some constant $C, \hat{g}^{(2)}=g^{(2)}$ with high probability. Therefore, $\hat{g}^{(2)}=g^{(2)}$ with high probability.

Finally, when $\hat{g}^{(2)}=g^{(2)}$, it is $\pi_{0} / 3$-proper with high probability according to Lemma 7 . Then by Lemma 5 again, $\hat{g}=g$ with high probability when $\alpha_{n}>C^{\prime} \log n / n$ for another large enough constant $C^{\prime}$.

Proof of Lemma 5. Suppose $\alpha_{n}=a \log n / n$ for some constant $a$. If for all nodes $v \in \mathcal{V}_{2}$, for some constant $\delta>0$,

$$
\left\|\frac{\hat{h}_{v}}{\left\|\hat{h}_{v}\right\|}-\frac{\tilde{B}\left(g_{v}, \cdot\right)}{\left\|\tilde{B}\left(g_{v}, \cdot\right)\right\|}\right\| \leq \delta,
$$

then we have the following separation conditions

$$
\begin{aligned}
& \sup _{v, v^{\prime} \in \mathcal{V}_{2}, g_{v}=g_{v^{\prime}}}\left\|\frac{\hat{h}_{v}}{\left\|\hat{h}_{v}\right\|}-\frac{\hat{h}_{v^{\prime}}}{\left\|\hat{h}_{v^{\prime}}\right\|}\right\| \leq 2 \delta, \\
& \inf _{v, v^{\prime} \in \mathcal{V}_{2}, g_{v} \neq g_{v^{\prime}}}\left\|\frac{\hat{h}_{v}}{\left\|\hat{h}_{v}\right\|}-\frac{\hat{h}_{v^{\prime}}}{\left\|\hat{h}_{v^{\prime}}\right\|}\right\| \geq \inf _{1 \leq k<k^{\prime} \leq K}\left\|\frac{\tilde{B}(k, \cdot)}{\|\tilde{B}(k, \cdot)\|}-\frac{\tilde{B}\left(k^{\prime}, \cdot\right)}{\left\|\tilde{B}\left(k^{\prime}, \cdot\right)\right\|}\right\|-2 \delta .
\end{aligned}
$$

We know from Lemma 8 that

$$
\inf _{1 \leq k<k^{\prime} \leq K}\left\|\frac{\tilde{B}(k, \cdot)}{\|\tilde{B}(k, \cdot)\|}-\frac{\tilde{B}\left(k^{\prime}, \cdot\right)}{\left\|\tilde{B}\left(k^{\prime}, \cdot\right)\right\|}\right\| \geq \psi_{0} \tilde{\gamma} .
$$

Thus the distance based clustering subroutine $\mathcal{D}$ used in Algorithm 1', such as the minimum spanning tree, can correctly cluster all nodes, i.e., $\hat{g}^{(2)}=g^{(2)}$, if

$$
\sup _{v, v^{\prime} \in \mathcal{V}_{2}, g_{v}=g_{v^{\prime}}}\left\|\frac{\hat{h}_{v}}{\left\|\hat{h}_{v}\right\|}-\frac{\hat{h}_{v^{\prime}}}{\left\|\hat{h}_{v^{\prime}}\right\|}\right\|<\inf _{v, v^{\prime} \in \mathcal{V}_{2}, g_{v} \neq g_{v^{\prime}}}\left\|\frac{\hat{h}_{v}}{\left\|\hat{h}_{v}\right\|}-\frac{\hat{h}_{v^{\prime}}}{\left\|\hat{h}_{v^{\prime}}\right\|}\right\| .
$$

Therefore, we only need to show that with high probability, $\left\|\frac{\hat{h}_{v}}{\left\|\hat{h}_{v}\right\|}-\frac{\tilde{B}\left(g_{v}, \cdot\right)}{\left\|\tilde{B}\left(g_{v}, \cdot\right)\right\|}\right\| \leq \delta$ for all nodes $v \in \mathcal{V}_{2}$, where

$$
0<\delta<\frac{\psi_{0} \tilde{\gamma}}{4}
$$

By Lemma 9, the approximation bound (7) and inequality (8) hold with high probability if we choose $a$ to be a constant larger than $4 q_{0} /\left(\psi_{0} \tilde{\gamma}\right)$, where $q_{0}=q_{0}\left(\pi_{0}, \psi_{0}, B_{0}, \tilde{\gamma} \psi_{0} / 4\right)$ as specified in Lemma 9. 
Lemma 8 (Lower bound of the distances between normalized rows of $\tilde{B}$ ). If a degree corrected block model satisfies Assumptions A1' and A4, and $\tilde{B}$ is defined as in equation (6), then

$$
\min _{1<k<k^{\prime} \leq K}\left\|\frac{\tilde{B}(k, \cdot)}{\|\tilde{B}(k, \cdot)\|}-\frac{\tilde{B}\left(k^{\prime}, \cdot\right)}{\left\|\tilde{B}\left(k^{\prime}, \cdot\right)\right\|}\right\| \geq \psi_{0} \tilde{\gamma} .
$$

Proof. Define matrix

$$
\Psi=\operatorname{diag}\left(\frac{\sum_{v^{\prime} \in \mathcal{I}_{1}^{(1)}} \psi_{v^{\prime}}}{\left|\mathcal{I}_{1}^{(1)}\right|}, \ldots, \frac{\sum_{v^{\prime} \in \mathcal{I}_{K}^{(1)}} \psi_{v^{\prime}}}{\left|\mathcal{I}_{K}^{(1)}\right|}\right)
$$

We only need to prove that $\left\|\frac{\Psi B_{0}(k, \cdot)^{T}}{\left\|\Psi B_{0}(k, \cdot)^{T}\right\|}-\frac{\Psi B_{0}\left(k^{\prime}, \cdot\right)^{T}}{\left\|\Psi B_{0}\left(k^{\prime}, \cdot\right)^{T}\right\|}\right\| \geq \psi_{0} \tilde{\gamma}$, for any $k \neq k^{\prime}$.

Now we define

$$
w=\frac{B_{0}(k, \cdot)^{T}}{\left\|\Psi B_{0}(k, \cdot)^{T}\right\|}-\frac{B_{0}\left(k^{\prime}, \cdot\right)^{T}}{\left\|\Psi B_{0}\left(k^{\prime}, \cdot\right)^{T}\right\|}=u / s-v / t
$$

where $u=\frac{B_{0}(k, \cdot)^{T}}{\left\|B_{0}(k, \cdot)^{T}\right\|}, v=\frac{B_{0}\left(k^{\prime}, \cdot\right)^{T}}{\left\|B_{0}\left(k^{\prime}, \cdot\right)^{T}\right\|}, s=\frac{\left\|\Psi B_{0}(k, \cdot)^{T}\right\|}{\left\|B_{0}(k, \cdot)^{T}\right\|}$, and $t=\frac{\left\|\Psi B_{0}\left(k^{\prime}, \cdot\right)^{T}\right\|}{\left\|B_{0}\left(k^{\prime}, \cdot\right)^{T}\right\|}$. By Assumption A4, we have

$$
\psi_{0} \leq \frac{\left\|\Psi B_{0}(k, \cdot)^{T}\right\|}{\left\|B_{0}(k, \cdot)^{T}\right\|} \leq 1, \quad \forall k
$$

Thus,

$$
\|w\| \geq \min _{\psi_{0} \leq s, t \leq 1}\left\|\frac{u}{s}-\frac{v}{t}\right\|
$$

Because $u$ and $v$ are two unit vectors with $u^{T} v \geq 0$, it is straightforward to check that the function

$$
f(t, s)=\left\|\frac{u}{s}-\frac{v}{t}\right\|^{2}=\frac{1}{t^{2}}+\frac{1}{s^{2}}-\frac{2}{t s} u^{T} v, \quad \psi_{0} \leq t, s \leq 1
$$

reaches its minimum $\|u-v\|^{2}$, when $t=s=1$. Therefore,

$$
\|w\| \geq\|u-v\|=\left\|\frac{B_{0}(k, \cdot)^{T}}{\left\|B_{0}(k, \cdot)^{T}\right\|}-\frac{B_{0}\left(k^{\prime}, \cdot\right)^{T}}{\left\|B_{0}\left(k^{\prime}, \cdot\right)^{T}\right\|}\right\| \geq \tilde{\gamma} .
$$

Using the fact that smallest eigenvalue of $\Psi$ satisfies $\lambda_{\min }(\Psi) \geq \psi_{0}$, we have

$$
\left\|\frac{\Psi B_{0}(k, \cdot)^{T}}{\left\|\Psi B_{0}(k, \cdot)^{T}\right\|}-\frac{\Psi B_{0}\left(k^{\prime}, \cdot\right)^{T}}{\left\|\Psi B_{0}\left(k^{\prime}, \cdot\right)^{T}\right\|}\right\|=\|\Psi w\| \geq \psi_{0}\|w\| \geq \psi_{0} \tilde{\gamma}
$$


Lemma 9. Given $\mathcal{V}_{1}, \mathcal{V}_{2}, g^{(1)}$, and $\hat{g}^{(1)}$ satisfying the conditions of Lemma 5 , let $\alpha_{n}=$ $(q / \delta) \log n / n$. For any $\delta>0$, there exists $q_{0}=q_{0}\left(\pi_{0}, \psi_{0}, B_{0}, \delta\right)$, such that if $q \geq q_{0}$,

$$
\mathbb{P}\left(\left\|\frac{\hat{h}_{v}}{\left\|\hat{h}_{v}\right\|}-\frac{\tilde{B}\left(g_{v}, \cdot\right)}{\left\|\tilde{B}\left(g_{v}, \cdot\right)\right\|}\right\| \leq \delta, \quad \forall v \in \mathcal{V}_{2}\right)=1-O\left(n^{-1}\right)
$$

where the probability is conditional on $\mathcal{V}_{1}, \mathcal{V}_{2}$ and $\hat{g}^{(1)}$.

Proof. Let $a=q / \delta$ and $L=\min _{k}\left\|B_{0}(k, \cdot)\right\|>0$. First, by the definition of $\tilde{B}$, we have

$$
\max \left\{\left\|\hat{h}_{v}\right\|,\left\|\psi_{v} \tilde{B}\left(g_{v}, \cdot\right)\right\|\right\} \geq\left\|\psi_{v} \tilde{B}\left(g_{v}, \cdot\right)\right\| \geq \psi_{0}^{2} \alpha_{n} \min _{j}\left\|B_{0}(j, \cdot)\right\|=\frac{\psi_{0}^{2} L q}{\delta} \frac{\log n}{n},
$$

Therefore,

$$
\begin{aligned}
\left\|\frac{\hat{h}_{v}}{\left\|\hat{h}_{v}\right\|}-\frac{\tilde{B}\left(g_{v}, \cdot\right)}{\left\|\tilde{B}\left(g_{v}, \cdot\right)\right\|}\right\| & =\left\|\frac{\hat{h}_{v}}{\left\|\hat{h}_{v}\right\|}-\frac{\psi_{v} \tilde{B}\left(g_{v}, \cdot\right)}{\left\|\psi_{v} \tilde{B}\left(g_{v}, \cdot\right)\right\|}\right\| \\
& \leq 2 \frac{\left\|\hat{h}_{v}-\psi_{v} \tilde{B}\left(g_{v}, \cdot\right)\right\|}{\max \left\{\left\|\hat{h}_{v}\right\|,\left\|\psi_{v} \tilde{B}\left(g_{v}, \cdot\right)\right\|\right\}} \\
& \leq \frac{2 \delta}{\psi_{0}^{2} L q} \frac{n}{\log n}\left\|\hat{h}_{v}-\psi_{v} \tilde{B}\left(g_{v}, \cdot\right)\right\| .
\end{aligned}
$$

So we only need to bound

$$
\mathbb{P}\left(\left\|\hat{h}_{v}-\psi_{v} \tilde{B}\left(g_{v}, \cdot\right)\right\| \geq \frac{\psi_{0}^{2} L q}{2} \frac{\log n}{n}\right) \leq \sum_{k=1}^{K} \mathbb{P}\left(\left|\hat{h}_{v, k}-\psi_{v} \tilde{B}\left(g_{v}, k\right)\right| \geq \frac{\psi_{0}^{2} L q}{2 \sqrt{K}} \frac{\log n}{n}\right),
$$

and the rest of the proof follows by adapting that of Lemma 6 . The details are given below. Since inequalities (4), (5) in Lemma 6 still hold, for any $k$, we have

$$
\begin{aligned}
\left|\hat{h}_{v, k}-\psi_{v} \tilde{B}\left(g_{v}, k\right)\right| \leq & \left|\frac{\sum_{v^{\prime} \in \hat{\mathcal{I}}_{k}^{(1)}} A_{v, v^{\prime}}-\sum_{v^{\prime} \in \mathcal{I}_{k}^{(1)}} A_{v, v^{\prime}}}{\left|\hat{\mathcal{I}}_{k}^{(1)}\right|}\right|+\left|\frac{\sum_{v^{\prime} \in \mathcal{I}_{k}^{(1)}} A_{v, v^{\prime}}}{\left|\hat{\mathcal{I}}_{k}^{(1)}\right|}-\frac{\sum_{v^{\prime} \in \mathcal{I}_{k}^{(1)}} A_{v, v^{\prime}}}{\left|\mathcal{I}_{k}^{(1)}\right|}\right| \\
& +\left|\frac{\sum_{v^{\prime} \in \mathcal{I}_{k}^{(1)}} A_{v, v^{\prime}}}{\left|\mathcal{I}_{k}^{(1)}\right|}-\frac{\sum_{v^{\prime} \in \mathcal{I}_{k}^{(1)}} \psi_{v^{\prime}}}{\left|\mathcal{I}_{k}^{(1)}\right|} \psi_{v} B\left(g_{v}, k\right)\right| \\
\leq & \frac{\left|\sum_{v^{\prime} \in \hat{\mathcal{I}}_{k}^{(1)}} A_{v, v^{\prime}}-\sum_{v^{\prime} \in \mathcal{I}_{k}^{(1)}} A_{v, v^{\prime}}\right|}{\pi_{0} n / 2}+\frac{|| \mathcal{I}_{k}^{(1)}|-| \hat{\mathcal{I}}_{k}^{(1)}||}{\pi_{0}^{2} n^{2} / 2} \sum_{v^{\prime} \in \mathcal{I}_{k}^{(1)}} A_{v, v^{\prime}} \\
& +\left|\frac{\sum_{v^{\prime} \in \mathcal{I}_{k}^{(1)}} A_{v, v^{\prime}}}{\left|\mathcal{I}_{k}^{(1)}\right|}-\frac{\sum_{v^{\prime} \in \mathcal{I}_{k}^{(1)}} \psi_{v^{\prime}}}{\left|\mathcal{I}_{k}^{(1)}\right|} \psi_{v} B\left(g_{v}, k\right)\right| \\
= & T_{1}+T_{2}+T_{3} .
\end{aligned}
$$


Now we only need to bound the three terms individually. First by (4) we know $\mid\left\{v^{\prime}: \hat{g}_{v^{\prime}}^{(1)} \neq\right.$ $\left.g_{v^{\prime}}^{(1)}\right\} \mid \leq n / f\left(\pi_{0} a \log n\right)$. Then using Bernstein's inequality we have for $n$ large enough,

$$
\begin{aligned}
\mathbb{P}\left(T_{1} \geq \frac{\psi_{0}^{2} L q}{6 \sqrt{K}} \frac{\log n}{n}\right) & \leq \mathbb{P}\left(\sum_{v^{\prime}: \hat{g}_{v^{\prime}}^{(1)} \neq g_{v^{\prime}}^{(1)}} A_{v, v^{\prime}} \geq \frac{\pi_{0} n}{2} \frac{\psi_{0}^{2} L q}{6 \sqrt{K}} \frac{\log n}{n}\right) \\
& \leq n^{-\frac{c_{1}^{\prime 2}\left(\pi_{0}, \psi_{0}, K, L\right) q^{2}}{a / f\left(\pi_{0} a \log n\right)+c_{1}^{\prime}\left(\pi_{0}, \psi_{0}, K, L\right) q / 3}} \leq n^{-c_{1}^{\prime}\left(\pi_{0}, \psi_{0}, K, L\right) q}
\end{aligned}
$$

where $c_{1}^{\prime}\left(\pi_{0}, \psi_{0}, K, L\right)$ is a positive constant depending only on $\pi_{0}, \psi_{0}, K$ and $L$.

To control $T_{2}$, similarly we have, for $n$ large enough, using Bernstein's inequality,

$$
\begin{aligned}
\mathbb{P}\left(T_{2}\right. & \left.\geq \frac{\psi_{0}^{2} L q}{6 \sqrt{K}} \frac{\log n}{n}\right) \leq \mathbb{P}\left(\sum_{v^{\prime} \in \mathcal{I}_{k}^{(1)}} A_{v, v^{\prime}} \geq \frac{\pi_{0}^{2} n^{2}}{2|| \mathcal{I}_{k}^{(1)}|-| \hat{\mathcal{I}}_{k}^{(1)}||} \frac{\psi_{0}^{2} L q}{6 \sqrt{K}} \frac{\log n}{n}\right) \\
& \leq \mathbb{P}\left(\sum_{v^{\prime} \in \mathcal{I}_{k}^{(1)}} A_{v, v^{\prime}} \geq \frac{L \psi_{0}^{2} \pi_{0}^{2} q \log n f\left(\pi_{0} a \log n\right)}{12 \sqrt{K}}\right) \\
& \leq n^{-\frac{c_{2}^{\prime 2}\left(\pi_{0}, \psi_{0}, K, L\right) f^{2}\left(\pi_{0} a \log n\right) q^{2}}{a+c_{2}^{\prime}\left(\pi_{0}, \psi_{0}, K, L\right) f\left(\pi_{0} a \log n\right) q / 3}} \leq n^{-c_{2}^{\prime}\left(\pi_{0}, \psi_{0}, K, L\right) q},
\end{aligned}
$$

where $c_{2}^{\prime}\left(\pi_{0}, \psi_{0}, K, L\right)$ is another positive constant function of $\pi_{0}, \psi_{0}, K$ and $L$.

Directly applying Bernstein's inequality to $T_{3}$, we have

$$
\begin{aligned}
\mathbb{P}\left(T_{3}>\frac{\psi_{0}^{2} L q}{6 \sqrt{K}} \frac{\log n}{n}\right) & \leq \mathbb{P}\left(\left|\sum_{v^{\prime} \in \mathcal{I}_{k}^{(1)}}\left[A_{v, v^{\prime}}-\psi_{v} \psi_{v^{\prime}} B\left(g_{v}, k\right)\right]\right| \geq \frac{\psi_{0}^{2} L q}{6 \sqrt{K}} \frac{\log n}{n} \pi_{0} n\right) \\
& \leq 2 n^{-c_{3}^{\prime}\left(\pi_{0}, \psi_{0}, K, L, \delta\right) q},
\end{aligned}
$$

where $c_{3}^{\prime}\left(\pi_{0}, \psi_{0}, K, L, \delta\right)$ is also a positive constant function independent of $n$.

The claimed result follows by choosing $q$ large enough and applying union bound over all $v \in \mathcal{V}_{2}$.

\section{References}

Abbe, E., Bandeira, A. S., \& Hall, G. (2014). Exact recovery in the stochastic block model. arXiv preprint arXiv:1405.3267. 
Adamic, L. A., \& Glance, N. (2005). The political blogosphere and the 2004 us election: divided they blog. In Proceedings of the 3rd international workshop on Link discovery, (pp. 36-43). ACM.

Anandkumar, A., Ge, R., Hsu, D., \& Kakade, S. M. (2014). A tensor approach to learning mixed membership community models. Journal of Machine Learning Research, 15, 22392312 .

Bickel, P. J., \& Chen, A. (2009). A nonparametric view of network models and newmangirvan and other modularities. Proceedings of the National Academy of Sciences, 106(50), 21068-21073.

Chaudhuri, K., Chung, F., \& Tsiatas, A. (2012). Spectral clustering of graphs with general degrees in the extended planted partition model. JMLR: Workshop and Conference Proceedings, 2012, 35.1-35.23.

Chen, Y., Sanghavi, S., \& Xu, H. (2012). Clustering sparse graphs. In P. Bartlett, F. Pereira, C. Burges, L. Bottou, \& K. Weinberger (Eds.) Advances in Neural Information Processing Systems 25, (pp. 2213-2221).

Coja-Oghlan, A. (2010). Graph partitioning via adaptive spectral techniques. Combinatorics, Probability and Computing, 19, 227-284.

Decelle, A., Krzakala, F., Moore, C., \& Zdeborová, L. (2011). Asymptotic analysis of the stochastic block model for modular networks and its algorithmic applications. Physical Review E, 84(6), 066106.

Faust, K., \& Wasserman, S. (1992). Blockmodels: Interpretation and evaluation. Social networks, 14(1), 5-61.

Fishkind, D. E., Sussman, D. L., Tang, M., Vogelstein, J. T., \& Priebe, C. E. (2013). Consistent adjacency-spectral partitioning for the stochastic block model when the model parameters are unknown. SIAM Journal on Matrix Analysis and Applications, 34 (1), $23-39$.

Holland, P. W., Laskey, K. B., \& Leinhardt, S. (1983). Stochastic blockmodels: First steps. Social networks, 5(2), 109-137.

Jin, J. (2012). Fast community detection by SCORE. arXiv:1211.5803.

Karrer, B., \& Newman, M. E. (2011). Stochastic blockmodels and community structure in networks. Physical Review E, 83(1), 016107.

Kemp, C., Tenenbaum, J. B., Griffiths, T. L., Yamada, T., \& Ueda, N. (2006). Learning systems of concepts with an infinite relational model. In $A A A I$, vol. 3, (p. 5). 
Le, C. M., Levina, E., \& Vershynin, R. (2014). Optimization via low-rank approximation, with applications to community detection in networks. arXiv preprint arXiv:1406.0067.

Lei, J., \& Rinaldo, A. (2013). Consistency of spectral clustering in sparse stochastic block models. arXiv preprint arXiv:1312.2050.

Massoulie, L. (2013). Community detection thresholds and the weak ramanujan property. arXiv preprint arXiv:1311.3085.

McSherry, F. (2001). Spectral partitioning of random graphs. In Foundations of Computer Science, 2001. Proceedings. 42nd IEEE Symposium on, (pp. 529-537). IEEE.

Mossel, E., Neeman, J., \& Sly, A. (2013). A proof of the block model threshold conjecture. arXiv preprint arXiv:1311.4115.

Newman, M. E., \& Girvan, M. (2004). Finding and evaluating community structure in networks. Physical review E, 69(2), 026113.

Rohe, K., Chatterjee, S., \& Yu, B. (2011). Spectral clustering and the high-dimensional stochastic blockmodel. The Annals of Statistics, 39, 1878-1915.

Skala, M. (2013). Hypergeometric tail inequalities: ending the insanity. arXiv preprint arXiv:1311.5939.

$\mathrm{Vu}$, V. (2014). A simple svd algorithm for finding hidden partitions. arXiv preprint arXiv:1404.3918.

Yan, X., Shalizi, C., Jensen, J. E., Krzakala, F., Moore, C., Zdeborová, L., Zhang, P., \& Zhu, Y. (2014). Model selection for degree-corrected block models. Journal of Statistical Mechanics: Theory and Experiment, 2014(5), P05007.

Zhao, Y., Levina, E., \& Zhu, J. (2012). Consistency of community detection in networks under degree-corrected stochastic block models. The Annals of Statistics, 40(4), 22662292 . 